УДК 517.5

O. M. Mulyava, M. M. Sheremeta

\title{
RELATIVE GROWTH OF DIRICHLET SERIES
}

O. M. Mulyava, M. M. Sheremeta. Relative growth of Dirichlet series, Mat. Stud. 49 (2018), 158-164.

Let $F$ and $G$ be entire functions given by Dirichlet series with exponents increasing to $+\infty$. In the term of a generalized order it is introduced a concept of their relative growth and its connection with the growth of $F$ and the growth of $G$ is shown.

1. Introduction. For an entire transcendental function $f(z)=\sum_{n=0}^{\infty} f_{n} z^{n}$ let $M_{f}(r)=\max \{|f(z)|:|z|=r\}$. The classical order of $f$ is defined as follows $\varrho[f]=$ $\inf \left\{\varrho>0: \ln M_{f}(r)<r^{\varrho}\right.$ for all large $\left.r\right\}$, i. e. $\varrho[f]=\varlimsup_{r \rightarrow+\infty} \frac{\ln \ln M_{f}(r)}{\ln r}$. Similarly $\lambda[f]=$ $\varliminf_{r \rightarrow+\infty} \frac{\ln \ln M_{f}(r)}{\ln r}$ is the lower order. We say that the function $f$ has a regular growth if $\lambda[f]=\varrho[f]$.

Suppose that $f$ and $g$ are entire transcendental functions. Then the functions $M_{f}(r)$ and $M_{g}(r)$ are continuous and increasing to $+\infty$ on $[0,+\infty)$. Therefore, there exists the function $M_{g}^{-1}(x)$ inverse to $M_{g}(r)$, which is continuous and increasing to $+\infty$ on $(|g(0)|,+\infty)$. The quantity $\varrho_{g}[f]=\inf \left\{\varrho>0: M_{f}(r)<M_{g}\left(r^{\varrho}\right)\right.$ for all large $\left.r\right\}$ is called the order of the function $f$ with respect to the function $g$. It is clear that $\varrho_{g}[f]=\varlimsup_{r \rightarrow+\infty} \frac{\ln M_{g}^{-1}\left(M_{f}(r)\right)}{\ln r}$. Similarly $\lambda_{g}[f]=\varliminf_{r \rightarrow+\infty} \frac{\ln M_{g}^{-1}\left(M_{f}(r)\right)}{\ln r}$ is called the lower order of $f$ with respect to $g$. If $\lambda_{g}[f]=\varrho_{g}[f]$ then we say that $f$ has a regular growth with respect to $g$. We remark that if $g(z)=e^{z}$ then $\varrho_{g}[f]=\varrho[f]$ and $\lambda_{g}[f]=\lambda[f]$.

Ch. Roy [1] tried to prove the following two theorems.

Theorem A. If $\varrho[f]<+\infty$ and $\varrho[g]>0$ then $\varrho_{g}[f] \geq \varrho[f] / \varrho[g]$, and if, moreover, $g$ has the regular growth then $\varrho_{g}[f]=\varrho[f] / \varrho[g]$.

Theorem B. If the function $f$ has the order $\varrho[f]<+\infty$ and the function $g(z)=\sum_{n=0}^{\infty} g_{n} z^{n}$ has the order $\varrho[g]>0$ and the regular growth then

$$
\varrho_{g}[f]=\varlimsup_{n \rightarrow \infty} \frac{\ln \left|g_{n}\right|}{\ln \left|f_{n}\right|} .
$$

2010 Mathematics Subject Classification: 33B50.

Keywords: Dirichlet series, generalized order, relative growth. doi:10.15330/ms.49.2.158-164 
In the proof of Theorem A in [1] it is used the estimate $\ln M_{g}(r)<r^{\varrho[g]+\varepsilon}$ for each $\varepsilon>0$ and all $r \geq r_{0}(\varepsilon)$. To obtain this estimate it is necessary that $\varrho[g]<+\infty$, but not $\varrho[g]>0$. Therefore, the conclusion of Theorem $\mathrm{A}$ is uncertain. In fact, in Theorem $\mathrm{A}$ it is necessary to replace the assumptions $\varrho[f]<+\infty$ and $\varrho[g]>0$ by the following one: neither $\varrho[f]=\varrho[g]=0$ nor $\varrho[f]=\varrho[g]=+\infty$ holds.

The conclusion of Theorem B is false. Indeed, the functions $f(z)=\sin z$ and $g(z)=\cos z$ have order 1 and the regular growth and by $(1) \varrho_{g}(f)=1$. But $\lim _{k \rightarrow \infty} \frac{\ln \left|g_{2 k}\right|}{\ln \left|f_{2 k}\right|}=+\infty$ and $\lim _{k \rightarrow \infty} \frac{\ln \left|g_{2 k+1}\right|}{\ln \left|f_{2 k+1}\right|}=0$. The conclusion of Theorem B is correct under the additional condition $\left|g_{n} / g_{n+1}\right| \nearrow+\infty$ as $n_{0} \leq n \rightarrow \infty$.

The aim of our note is to prove analogues of Theorems A and B for entire Dirichlet series of finite generalized orders.

2. Main results. Let $\Lambda=\left(\lambda_{n}\right)$ be a sequence of nonnegative numbers increasing to $+\infty$ and $S(\Lambda)$ the class of entire Dirichlet series

$$
F(s)=\sum_{n=1}^{\infty} f_{n} \exp \left\{s \lambda_{n}\right\}, \quad s=\sigma+i t .
$$

For $F \in S(\Lambda)$ we denote $M_{F}(\sigma)=\sup \{|F(\sigma+i t)|: t \in \mathbb{R}\}$.

Let $L$ be the class of continuous increasing functions $\alpha$ such that $\alpha(x) \geq 0$ for $x \geq x_{0}$, $\alpha(x)=\alpha\left(x_{0}\right)$ for $x \leq x_{0}$, and on $\left[x_{0},+\infty\right)$ the function $\alpha$ increases to $+\infty$. We say that $\alpha \in L^{0}$ if $\alpha \in L$ and $\alpha(x(1+o(1)))=(1+o(1)) \alpha(x)$ as $x \rightarrow+\infty$; further, $\alpha \in L_{s i}$ if $\alpha \in L$ and for any $c>0 \alpha(c x)=(1+o(1)) \alpha(x)$ as $x \rightarrow+\infty$. It is easy to see that $L_{s i} \subset L^{0}$. Functions from $L_{s i}$ are called slowly increasing.

For $\alpha \in L$ and $\beta \in L$

$$
\varrho_{\alpha, \beta}[F]=\varlimsup_{\sigma \rightarrow+\infty} \frac{\alpha\left(\ln M_{F}(\sigma)\right)}{\beta(\sigma)}, \quad \lambda_{\alpha, \beta}[F]=\varliminf_{\sigma \rightarrow+\infty} \frac{\alpha\left(\ln M_{F}(\sigma)\right)}{\beta(\sigma)} .
$$

are called the generalized order and the lower generalized order of $G$, respective. We say that the function $F$ has generalized regular growth if $\varrho_{\alpha, \beta}[F]=\lambda_{\alpha, \beta}[F]$. We need the following lemma ([2]).

Lemma 1. Let $\alpha \in L$ and $\beta \in L$ be continuously differentiable functions, $0<p<+\infty$ and one of next conditions holds:

a) $\alpha \in L^{0}, \beta(\ln x) \in L^{0}, \frac{d \beta^{-1}(c \alpha(x))}{d \ln x} \rightarrow \frac{1}{p}(x \rightarrow+\infty)$ for each $c \in(0,+\infty)$ and $\ln n=o\left(\lambda_{n}\right)$ $(n \rightarrow \infty)$

b) $\alpha \in L_{s i}, \beta \in L^{0}, \frac{d \beta^{-1}(c \alpha(x))}{d \ln x}=O(1)(x \rightarrow+\infty)$ and $\ln n=o\left(\lambda_{n} \beta^{-1}\left(c \alpha\left(\lambda_{n}\right)\right)\right)(n \rightarrow \infty)$ for each $c \in(0,+\infty)$.

Then

$$
\varrho_{\alpha, \beta}[F]=\varlimsup_{n \rightarrow \infty} \frac{\alpha\left(\lambda_{n} / p\right)}{\beta\left(\frac{1}{p}+\frac{1}{\lambda_{n}} \ln \frac{1}{\left|a_{n}\right|}\right)},
$$

and if, moreover, $\alpha\left(\lambda_{n+1} / p\right)=(1+o(1)) \alpha\left(\lambda_{n} / p\right)$ and $\frac{\ln \left|a_{n}\right|-\ln \left|a_{n+1}\right|}{\lambda_{n+1}-\lambda_{n}} \quad \nearrow+\infty$ as $n_{0} \leq n \rightarrow \infty$ then

$$
\lambda_{\alpha, \beta}[F]=\varliminf_{n \rightarrow \infty} \frac{\alpha\left(\lambda_{n} / p\right)}{\beta\left(\frac{1}{p}+\frac{1}{\lambda_{n}} \ln \frac{1}{\left|a_{n}\right|}\right)} .
$$


We remark that the generalized order can be define as follows $\varrho_{\alpha, \beta}[F]=\inf \{\varrho>0$ : $\ln M(\sigma) \leq \alpha^{-1}(\varrho \beta(\sigma))$ for all $\left.\sigma \geq \sigma_{0}(\varrho)\right\}$. Therefore, by analogy we define the generalized order $\varrho_{\alpha, \beta}[F]_{G}$ of the function $F$ with respect to a function $G$, given by an entire Dirichlet series

$$
G(s)=\sum_{n=1}^{\infty} g_{n} \exp \left\{s \lambda_{n}\right\}
$$

as follows $\varrho_{\alpha, \beta}[F]_{G}=\inf \left\{\varrho>0: \alpha\left(\ln M_{F}(\sigma)\right) \leq \alpha\left(\ln M_{G}\left(\beta^{-1}(\varrho \beta(\sigma))\right)\right.\right.$ for all $\left.\sigma \geq \sigma_{0}(\varrho)\right\}$. Since the function $M_{G}(\sigma)$ is continuous and increasing to $+\infty$ on $(-\infty,+\infty)$, there exists the function $M_{G}^{-1}(x)$ inverse to $M_{G}(\sigma)$ which increases to $+\infty$ on $\left[x_{0},+\infty\right)$. Therefore,

$$
\varrho_{\alpha, \beta}[F]_{G}=\varlimsup_{\sigma \rightarrow+\infty} \frac{\beta\left(M_{G}^{-1}\left(M_{F}(\sigma)\right)\right)}{\beta(\sigma)} .
$$

Similarly we define the lower generalized order $\lambda_{\alpha, \beta}[F]_{G}$ of $F$ with respect to $G$ by the formula

$$
\lambda_{\alpha, \beta}[F]_{G}=\varliminf_{\sigma \rightarrow+\infty} \frac{\beta\left(M_{G}^{-1}\left(M_{F}(\sigma)\right)\right)}{\beta(\sigma)},
$$

and say that $F$ has generalized regular growth with respect to $G$ if $\varrho_{\alpha, \beta}[F]_{G}=\lambda_{\alpha, \beta}[F]_{G}$.

The following theorem establishes connections between $\varrho_{\alpha, \beta}[F], \varrho_{\alpha, \beta}[G]$ and $\varrho_{\alpha, \beta}[F]_{G}$ and between $\lambda_{\alpha, \beta}[F], \lambda_{\alpha, \beta}[G]$ and $\lambda_{\alpha, \beta}[F]_{G}$.

Theorem 1. Let $\alpha \in L$ and $\beta \in L$. Except for cases, when $\varrho_{\alpha, \beta}[F]=\varrho_{\alpha, \beta}[G]=0$ or $\varrho_{\alpha, \beta}[F]=\varrho_{\alpha, \beta}[G]=+\infty$, the inequality $\varrho_{\alpha, \beta}[F]_{G} \geq \varrho_{\alpha, \beta}[F] / \varrho_{\alpha, \beta}[G]$ is true and subject to the condition of the generalized regular growth of $G$ this inequality converts into an equality.

Except for cases, when $\lambda_{\alpha, \beta}[F]=\lambda_{\alpha, \beta}[G]=0$ or $\lambda_{\alpha, \beta}[F]=\lambda_{\alpha, \beta}[G]=+\infty$, the inequality $\lambda_{\alpha, \beta}[F]_{G} \leq \lambda_{\alpha, \beta}[F] / \lambda_{\alpha, \beta}[G]$ is true and subject to the condition of the generalized regular growth of $G$ this inequality converts into an equality.

Proof. Indeed,

$$
\begin{gathered}
\varrho_{\alpha, \beta}[F]_{G}=\varlimsup_{x \rightarrow+\infty} \frac{\beta\left(M_{G}^{-1}(x)\right.}{\beta\left(M_{F}^{-1}(x)\right)}=\varlimsup_{x \rightarrow+\infty} \frac{\alpha(\ln x)}{\beta\left(M_{F}^{-1}(x)\right)} \frac{\beta\left(M_{G}^{-1}(x)\right)}{\alpha(\ln x)} \geq \\
\geq \varlimsup_{x \rightarrow+\infty} \frac{\alpha(\ln x)}{\beta\left(M_{F}^{-1}(x)\right)} \varliminf_{x \rightarrow+\infty} \frac{\beta\left(M_{G}^{-1}(x)\right)}{\alpha(\ln x)}=\varlimsup_{\sigma \rightarrow+\infty} \frac{\alpha\left(\ln M_{F}(\sigma)\right)}{\beta(\sigma)} \varliminf_{\sigma \rightarrow+\infty} \frac{\beta(\sigma)}{\alpha\left(\ln M_{G}(\sigma)\right)}=\frac{\varrho_{\alpha, \beta}[F]}{\varrho_{\alpha, \beta}[G]}
\end{gathered}
$$

and if there exists $\lim _{\sigma \rightarrow+\infty} \alpha\left(\ln M_{G}(\sigma)\right) / \beta(\sigma)=\lambda_{\alpha, \beta}[G]=\varrho_{\alpha, \beta}[G]$ then

$$
\varrho_{\alpha, \beta}[F]_{G}=\varlimsup_{x \rightarrow+\infty} \frac{\alpha(\ln x)}{\beta\left(M_{F}^{-1}(x)\right)} \frac{\beta\left(M_{G}^{-1}(x)\right)}{\alpha(\ln x)}=\varlimsup_{x \rightarrow+\infty} \frac{\alpha(\ln x)}{\beta\left(M_{F}^{-1}(x)\right)} \lim _{x \rightarrow+\infty} \frac{\beta\left(M_{G}^{-1}(x)\right)}{\alpha(\ln x)}=\frac{\varrho_{\alpha, \beta}[F]}{\varrho_{\alpha, \beta}[G]} .
$$

The first part of Theorem 1 is proved.

The proof of second part is similar. Indeed,

$$
\lambda_{\alpha, \beta}[F]_{G}=\varliminf_{x \rightarrow+\infty} \frac{\alpha(\ln x)}{\beta\left(M_{F}^{-1}(x)\right)} \frac{\beta\left(M_{G}^{-1}(x)\right)}{\alpha(\ln x)} \leq \varliminf_{x \rightarrow+\infty} \frac{\alpha(\ln x)}{\beta\left(M_{F}^{-1}(x)\right)} \varlimsup_{x \rightarrow+\infty} \frac{\beta\left(M_{G}^{-1}(x)\right)}{\alpha(\ln x)}=\frac{\lambda_{\alpha, \beta}[F]}{\lambda_{\alpha, \beta}[G]}
$$

and if there exists $\lim _{\sigma \rightarrow+\infty} \alpha\left(\ln M_{F}(\sigma)\right) / \beta(\sigma)=\lambda_{\alpha, \beta}[F]=\varrho_{\alpha, \beta}[F]$ then

$$
\lambda_{\alpha, \beta}[F]_{G}=\varlimsup_{x \rightarrow+\infty} \frac{\alpha(\ln x)}{\beta\left(M_{F}^{-1}(x)\right)} \lim _{x \rightarrow+\infty} \frac{\beta\left(M_{G}^{-1}(x)\right)}{\alpha(\ln x)}=\frac{\lambda_{\alpha, \beta}[F]}{\lambda_{\alpha, \beta}[G]} .
$$


We remark that except the cases, when $\varrho_{\alpha, \beta}[F]=\varrho_{\alpha, \beta}[G]=0$ or $\varrho_{\alpha, \beta}[F]=\varrho_{\alpha, \beta}[G]=+\infty$, the inequality $\varrho_{\alpha, \beta}[G]_{F} \geq \varrho_{\alpha, \beta}[G] / \varrho_{\alpha, \beta}[F]$ is also true. Therefore, $\varrho_{\alpha, \beta}[F]_{G} \cdot \varrho_{\alpha, \beta}[G]_{F} \geq 1$, and if the functions $F$ and $G$ have generalized regular growth then $\varrho_{\alpha, \beta}[F]_{G} \cdot \varrho_{\alpha, \beta}[G]_{F}=1$. A similar conclusion can be done relatively $\lambda_{\alpha, \beta}[F]_{G}$ and $\lambda_{\alpha, \beta}[G]_{F}$.

Remark also that if we define a generalized order $\varrho_{\alpha, \beta}^{*}[F]_{G}$ of the function $F$ with respect to $G$ as follows $\varrho_{\alpha, \beta}^{*}[F]_{G}=\inf \left\{\varrho>0: \alpha\left(\ln M_{F}(\sigma)\right) \leq \varrho \alpha\left(\ln M_{G}(\sigma)\right)\right.$ for all $\left.\sigma \geq \sigma_{0}(\varrho)\right\}$, i. e.

$$
\varrho_{\alpha, \beta}^{*}[F]_{G}=\varlimsup_{\sigma \rightarrow+\infty} \frac{\alpha\left(\ln M_{F}(\sigma)\right)}{\alpha\left(\ln M_{G}(\sigma)\right)},
$$

then, repeating the proof of Theorem 1 , we obtain the inequality $\varrho_{\alpha, \beta}^{*}[F]_{G} \geq \varrho_{\alpha, \beta}[F] / \varrho_{\alpha, \beta}[G]$ and subject to the condition of the generalized regular growth of $G$ we obtain also the equality $\varrho_{\alpha, \beta}^{*}[F]_{G}=\varrho_{\alpha, \beta}[F] / \varrho_{\alpha, \beta}[G]$. Hence it follows that if $G$ has generalized regular growth then $\varrho_{\alpha, \beta}^{*}[F]_{G}=\varrho_{\alpha, \beta}[F]_{G}$. This equality can be also obtain using the definitions of $\varrho_{\alpha, \beta}^{*}[F]_{G}$ and $\varrho_{\alpha, \beta}[F]_{G}$.

Using Lemma 1 now we prove the following theorem.

Theorem 2. Let entire Dirichlet series (2) and (3) have generalized orders $\varrho_{\alpha, \beta}[F], \varrho_{\alpha, \beta}[G]$ and lower orders $\lambda_{\alpha, \beta}[F], \lambda_{\alpha, \beta}[G]$. Suppose that $0<p<+\infty$, the functions $\alpha \in L$ and $\beta \in L$ are continuously differentiable and satisfy conditions either a) or b) of Lemma 1 and $\alpha\left(\lambda_{n+1} / p\right)=(1+o(1)) \alpha\left(\lambda_{n} / p\right)$ as $n \rightarrow \infty$. then

If the function $G$ has generalized regular growth and $\frac{\ln \left|g_{n}\right|-\ln \left|g_{n+1}\right|}{\lambda_{n+1}-\lambda_{n}} \nearrow+\infty$ as $n_{0} \leq n \rightarrow \infty$

$$
\varrho_{\alpha, \beta}[F]_{G}=\varkappa^{*}=: \varlimsup_{n \rightarrow \infty} \frac{\beta\left(\frac{1}{p}+\frac{1}{\lambda_{n}} \ln \frac{1}{\left|g_{n}\right|}\right)}{\beta\left(\frac{1}{p}+\frac{1}{\lambda_{n}} \ln \frac{1}{\left|f_{n}\right|}\right)}
$$

except for the cases, when $\varrho_{\alpha, \beta}[F]=\varrho_{\alpha, \beta}[G]=0$ or $\varrho_{\alpha, \beta}[F]=\varrho_{\alpha, \beta}[G]=+\infty$. then

Moreover, if $F$ has generalized regular growth and $\frac{\ln \left|f_{n}\right|-\ln \left|f_{n+1}\right|}{\lambda_{n+1}-\lambda_{n}} \nearrow+\infty$ as $n_{0} \leq n \rightarrow \infty$

$$
\lambda_{\alpha, \beta}[F]_{G}=\varkappa_{*}=: \varliminf_{n \rightarrow \infty} \frac{\beta\left(\frac{1}{p}+\frac{1}{\lambda_{n}} \ln \frac{1}{\left|g_{n}\right|}\right)}{\beta\left(\frac{1}{p}+\frac{1}{\lambda_{n}} \ln \frac{1}{\left|f_{n}\right|}\right)}
$$

except for the cases, when $\lambda_{\alpha, \beta}[F]=\lambda_{\alpha, \beta}[G]=0$ or $\lambda_{\alpha, \beta}[F]=\lambda_{\alpha, \beta}[G]=+\infty$.

Proof. By Theorem 1 and Lemma 1 we have

$$
\begin{aligned}
\varrho_{\alpha, \beta}[F]_{G}=\frac{\varrho_{\alpha, \beta}[F]}{\varrho_{\alpha, \beta}[G]} & =\varlimsup_{n \rightarrow \infty} \frac{\alpha\left(\lambda_{n} / p\right)}{\beta\left(\frac{1}{p}+\frac{1}{\lambda_{n}} \ln \frac{1}{\left|f_{n}\right|}\right)} \varliminf_{n \rightarrow \infty} \frac{\beta\left(\frac{1}{p}+\frac{1}{\lambda_{n}} \ln \frac{1}{\left|g_{n}\right|}\right)}{\alpha\left(\lambda_{n} / p\right)} \leq \\
& \leq \varlimsup_{n \rightarrow \infty} \frac{\beta\left(\frac{1}{p}+\frac{1}{\lambda_{n}} \ln \frac{1}{\left|g_{n}\right|}\right)}{\beta\left(\frac{1}{p}+\frac{1}{\lambda_{n}} \ln \frac{1}{\left|f_{n}\right|}\right)}=: \varkappa^{*} .
\end{aligned}
$$

On the other hand, let $\varkappa^{*}>0$. Then for every $\varepsilon \in\left(0, \varkappa^{*}\right)$ there exists an increasing to $\infty$ sequence $\left(n_{k}\right)$ of integers such that

$$
\beta\left(\frac{1}{p}+\frac{1}{\lambda_{n_{k}}} \ln \frac{1}{\left|g_{n_{k}}\right|}\right)>\left(\varkappa^{*}-\varepsilon\right) \beta\left(\frac{1}{p}+\frac{1}{\lambda_{n_{k}}} \ln \frac{1}{\left|f_{n_{k}}\right|}\right) .
$$


and, thus,

$$
\varlimsup_{n \rightarrow \infty} \frac{\alpha\left(\lambda_{n} / p\right)}{\beta\left(\frac{1}{p}+\frac{1}{\lambda_{n}} \ln \frac{1}{\left|f_{n}\right|}\right)}>\left(\varkappa^{*}-\varepsilon\right) \varliminf_{n \rightarrow \infty} \frac{\alpha\left(\lambda_{n} / p\right)}{\beta\left(\frac{1}{p}+\frac{1}{\lambda_{n}} \ln \frac{1}{\left|g_{n}\right|}\right)} .
$$

Since $\varrho_{\alpha, \beta}[G]=\lambda_{\alpha, \beta}[G]$, by Lemma 1 we obtain the inequality $\varrho_{\alpha, \beta}[F]>\left(\varkappa^{*}-\varepsilon\right) \varrho_{\alpha, \beta}[G]$, that is in view of arbitrariness of $\varepsilon$ the inequality $\varrho_{\alpha, \beta}[F]=\varrho_{\alpha, \beta}[F] / \varrho_{\alpha, \beta}[F] \geq \varkappa^{*}$ is true. For $\varkappa^{*}=0$ the last inequality is obvious. In view of (4) the first part of Theorem 2 is proved. For the proof of the second part we remark that since the function $G$ has generalized regular growth, by Theorem 1 and Lemma 1

$$
\begin{aligned}
\lambda_{\alpha, \beta}[F]_{G}=\frac{\lambda_{\alpha, \beta}[F]}{\lambda_{\alpha, \beta}[G]} & =\varliminf_{n \rightarrow \infty} \frac{\alpha\left(\lambda_{n} / p\right)}{\beta\left(\frac{1}{p}+\frac{1}{\lambda_{n}} \ln \frac{1}{\left|f_{n}\right|}\right)} \varlimsup_{n \rightarrow \infty} \frac{\beta\left(\frac{1}{p}+\frac{1}{\lambda_{n}} \ln \frac{1}{\left|g_{n}\right|}\right)}{\alpha\left(\lambda_{n} / p\right)} \geq \\
& \geq \varliminf_{n \rightarrow \infty} \frac{\beta\left(\frac{1}{p}+\frac{1}{\lambda_{n}} \ln \frac{1}{\left|g_{n}\right|}\right)}{\beta\left(\frac{1}{p}+\frac{1}{\lambda_{n}} \ln \frac{1}{\left|f_{n}\right|}\right)}=: \varkappa_{*} .
\end{aligned}
$$

On the other hand, if $\varkappa_{*}<+\infty$ then for every $\varepsilon \in\left(0, \varkappa^{*}\right)$ there exists an increasing to $\infty$ sequence $\left(n_{k}\right)$ of integers such that

$$
\beta\left(\frac{1}{p}+\frac{1}{\lambda_{n_{k}}} \ln \frac{1}{\left|g_{n_{k}}\right|}\right)<\left(\varkappa_{*}+\varepsilon\right) \beta\left(\frac{1}{p}+\frac{1}{\lambda_{n_{k}}} \ln \frac{1}{\left|f_{n_{k}}\right|}\right) .
$$

and, thus,

$$
\varlimsup_{n \rightarrow \infty} \frac{\alpha\left(\lambda_{n} / p\right)}{\beta\left(\frac{1}{p}+\frac{1}{\lambda_{n}} \ln \frac{1}{\left|f_{n}\right|}\right)}<\left(\varkappa_{*}+\varepsilon\right) \varliminf_{n \rightarrow \infty} \frac{\alpha\left(\lambda_{n} / p\right)}{\beta\left(\frac{1}{p}+\frac{1}{\lambda_{n}} \ln \frac{1}{\left|g_{n}\right|}\right)},
$$

whence, as above, in view of the generalized regular growth of $F$ we obtain the inequality $\lambda_{\alpha, \beta}[F]=\lambda_{\alpha, \beta}[F] / \lambda_{\alpha, \beta}[F] \leq \varkappa_{*}$. The last inequality is trivial if $\varkappa^{*}=+\infty$. By virtue of (5) the proof of Theorem 2 is completed.

3. Corollaries and addition. For $\beta(x) \equiv x\left(x \geq x_{0}\right)$ and $\alpha \in L_{s i}$ by $\varrho_{\alpha}[F], \lambda_{\alpha}[F], \varrho_{\alpha}[F]_{G}$ and $\lambda_{\alpha}[F]_{G}$ we denote $\varrho_{\alpha, \beta}[F], \lambda_{\alpha, \beta}[F], \varrho_{\alpha, \beta}[F]_{G}$ and $\lambda_{\alpha, \beta}[F]_{G}$, respective. In particular, if $\alpha(x) \equiv \ln x$ for $x \geq x_{0}$ then we obtain definitions of $R$-orders and lower $R$-orders.

Corollary 1. Let $\alpha \in L_{s i}, \frac{d \alpha(x)}{d \ln x}=O(1)$ as $x \rightarrow+\infty, \alpha\left(\lambda_{n+1}\right)=(1+o(1)) \alpha\left(\lambda_{n}\right)$ and $\ln n=o\left(\lambda_{n} \alpha\left(\lambda_{n}\right)\right)$ as $n \rightarrow \infty$. If the function $G$ has generalized regular growth and $\frac{\ln \left|g_{n}\right|-\ln \left|g_{n+1}\right|}{\lambda_{n+1}-\lambda_{n}} \nearrow+\infty$ as $n_{0} \leq n \rightarrow \infty$ then

$$
\varrho_{\alpha}[F]_{G}=\varlimsup_{n \rightarrow \infty} \frac{\ln \left(1 /\left|g_{n}\right|\right)}{\ln \left(1 /\left|f_{n}\right|\right)}
$$

except for the cases, when $\varrho_{\alpha}[F]=\varrho_{\alpha}[G]=0$ or $\varrho_{\alpha}[F]=\varrho_{\alpha}[G]=+\infty$.

Moreover, if $F$ has generalized regular growth and $\frac{\ln \left|f_{n}\right|-\ln \left|f_{n+1}\right|}{\lambda_{n+1}-\lambda_{n}} \nearrow+\infty$ as $n_{0} \leq n \rightarrow \infty$ then

$$
\lambda_{\alpha}[F]_{G}=\varliminf_{n \rightarrow \infty} \frac{\ln \left(1 /\left|g_{n}\right|\right)}{\ln \left(1 /\left|f_{n}\right|\right)}
$$

except the cases, when $\lambda_{\alpha}[F]=\lambda_{\alpha}[G]=0$ or $\lambda_{\alpha}[F]=\lambda_{\alpha}[G]=+\infty$. 
In this corollary the function $\alpha$ is absent on the right-hand side of formulas (6) and (7). A question arises: when is it possible to remove this function from the left-hand side of these formulas.

We put $\Phi(\sigma)=\ln M_{G}(\sigma)$. Then the function $\Phi$ is convex and continuously differentiable except the countable set of points, in which there exist one-sided derivatives and besides the left-sided derivative is less than the right-sided derivative. We remark that if $\frac{d \ln \Phi(\sigma)}{d \ln \sigma} \rightarrow+\infty$ as $\sigma \rightarrow+\infty$ and $\Phi^{-1}$ is the inverse function to $\Phi$ then $\frac{d \ln \Phi^{-1}(x)}{d \ln x} \rightarrow 0$ as $x \rightarrow+\infty$, that is $\Phi^{-1}$ is slowly increasing. We remark also that $\sigma=o\left(\ln M_{G}(\sigma)\right)$ as $\sigma \rightarrow+\infty$, i. e. $\Phi^{-1}(x)=o(x)$ as $x \rightarrow+\infty$. Finally, $\frac{\Phi^{-1}\left(\ln M_{G}(\sigma)\right)}{\sigma}=1$. Therefore, if we choose the function $\alpha \in L$ such that $\alpha(x)=\Phi^{-1}(x)$ then we get the following corollary.

Corollary 2. Suppose that Dirichlet series (2) and (3) are entire. Let $\Phi(\sigma)=\ln M_{G}(\sigma)$, $\frac{d \ln \Phi(\sigma)}{d \ln \sigma} \rightarrow+\infty$ as $\sigma \rightarrow+\infty, \Phi^{-1}\left(\lambda_{n+1}\right)=(1+o(1)) \Phi^{-1}\left(\lambda_{n}\right)$ and $\ln n=o\left(\lambda_{n} \Phi^{-1}\left(\lambda_{n}\right)\right)$ as $n \rightarrow \infty$. If $\frac{\ln \left|g_{n}\right|-\ln \left|g_{n+1}\right|}{\lambda_{n+1}-\lambda_{n}} \nearrow+\infty$ as $n_{0} \leq n \rightarrow \infty$ then

$$
\varlimsup_{\sigma \rightarrow+\infty} \frac{M_{G}^{-1}\left(M_{F}(\sigma)\right)}{\sigma}=\varlimsup_{n \rightarrow \infty} \frac{\ln \left(1 /\left|g_{n}\right|\right)}{\ln \left(1 /\left|f_{n}\right|\right)} .
$$

Moreover, if $F$ has generalized regular growth and $\frac{\ln \left|f_{n}\right|-\ln \left|f_{n+1}\right|}{\lambda_{n+1}-\lambda_{n}} \nearrow+\infty$ as $n_{0} \leq n \rightarrow \infty$ then

$$
\varliminf_{\sigma \rightarrow+\infty} \frac{M_{G}^{-1}\left(M_{F}(\sigma)\right)}{\sigma}=\varliminf_{n \rightarrow \infty} \frac{\ln \left(1 /\left|g_{n}\right|\right)}{\ln \left(1 /\left|f_{n}\right|\right)}
$$

Now we suppose that $F_{j}(1 \leq j \leq m)$ are entire functions given by Dirichlet series

$$
F_{j}(s)=\sum_{n=1}^{\infty} f_{n, j} \exp \left\{s \lambda_{n}\right\}
$$

In [3] the following theorem is proved.

Theorem C. Let $\alpha$ and $\beta$ be continuously differentiable functions, the condition $b$ ) of Lemma 1 hold, and all functions (8) have generalized regular growth. Suppose that $\alpha\left(\lambda_{n+1}\right)=$ $(1+o(1)) \alpha\left(\lambda_{n}\right)$ and $\frac{\ln \left|f_{n, j}\right|-\ln \left|f_{n+1, j}\right|}{\lambda_{n+1}-\lambda_{n}} \nearrow+\infty$ as $n_{0} \leq n \rightarrow \infty$ for each $j$. If

$$
\beta\left(\frac{1}{\lambda_{n}} \ln \frac{1}{\left|f_{n}\right|}\right)=(1+o(1)) \prod_{j=1}^{m} \beta\left(\frac{1}{\lambda_{n}} \ln \frac{1}{\left|f_{n, j}\right|}\right)^{\omega_{j}}, \quad n \rightarrow \infty,
$$

where $\omega_{j}>0$ and $\sum_{j=1}^{m} \omega_{j}=1$, then function (2) has generalized regular growth and $\varrho_{\alpha, \beta}[F]=\prod_{j=1}^{m}\left(\varrho_{\alpha, \beta}\left[F_{j}\right]\right)^{\omega_{j}}$.

Here we prove the following analogue of Theorem C.

Theorem 3. Let $\alpha$ and $\beta$ be continuously differentiable functions, the condition $b$ ) of Lemma 1 hold, and all functions (8) have generalized regular growth with respect to $G$. Suppose that $\alpha\left(\lambda_{n+1}\right)=(1+o(1)) \alpha\left(\lambda_{n}\right)$ and $\frac{\ln \left|a_{n, j}\right|-\ln \left|a_{n+1, j}\right|}{\lambda_{n+1}-\lambda_{n}} \nearrow+\infty$ as $n_{0} \leq n \rightarrow \infty$ for each $j$ and condition (9) holds.

If the function $G$ has generalized regular growth and $\frac{\ln \left|g_{n}\right|-\ln \left|g_{n+1}\right|}{\lambda_{n+1}-\lambda_{n}} \nearrow+\infty$ as $n_{0} \leq n \rightarrow$ $\infty$ then $\varrho_{\alpha, \beta}[F]_{G}=\prod_{j=1}^{m}\left(\varrho_{\alpha, \beta}\left[F_{j}\right]_{G}\right)^{\omega_{j}}$ except for cases, when $\varrho_{\alpha, \beta}\left[F_{j}\right]=\varrho_{\alpha, \beta}[G]=0$ or $\varrho_{\alpha, \beta}\left[F_{j}\right]=\varrho_{\alpha, \beta}[G]=+\infty$ for some $j$. 
Proof. Since functions (8) have generalized regular growth with respect to $G$, by Theorem 1

$$
\varrho_{\alpha, \beta}\left[F_{j}\right]_{G}=\lambda_{\alpha, \beta}\left[F_{j}\right]=\lim _{n \rightarrow \infty} \frac{\beta\left(\frac{1}{p}+\frac{1}{\lambda_{n}} \ln \frac{1}{\left|g_{n}\right|}\right)}{\beta\left(\frac{1}{p}+\frac{1}{\lambda_{n}} \ln \frac{1}{\left|f_{n, j}\right|}\right)},
$$

i. e. in view of (9) there exists

$$
\begin{gathered}
\lim _{n \rightarrow \infty} \frac{\beta\left(\frac{1}{p}+\frac{1}{\lambda_{n}} \ln \frac{1}{\left|f_{n}\right|}\right)}{\beta\left(\frac{1}{p}+\frac{1}{\lambda_{n}} \ln \frac{1}{\left|g_{n}\right|}\right)}=\lim _{n \rightarrow \infty} \frac{\prod_{j=1}^{m} \beta\left(\frac{1}{\lambda_{n}} \ln \frac{1}{\left|f_{n, j}\right|}\right)^{\omega_{j}}}{\beta\left(\frac{1}{p}+\frac{1}{\lambda_{n}} \ln \frac{1}{\left|g_{n}\right|}\right)}= \\
=\lim _{n \rightarrow \infty} \prod_{j=1}^{m}\left(\frac{\beta\left(\frac{1}{\lambda_{n}} \ln \frac{1}{\left|f_{n, j}\right|}\right)}{\beta\left(\frac{1}{p}+\frac{1}{\lambda_{n}} \ln \frac{1}{\left|g_{n}\right|}\right)}\right)^{\omega_{j}}=\prod_{j=1}^{m}\left(\lim _{n \rightarrow \infty} \frac{\beta\left(\frac{1}{\lambda_{n}} \ln \frac{1}{\left|f_{n, j}\right|}\right)}{\beta\left(\frac{1}{p}+\frac{1}{\lambda_{n}} \ln \frac{1}{\left|g_{n}\right|}\right)}\right)^{\omega_{j}}=\prod_{j=1}^{m}\left(\frac{1}{\varrho_{\alpha, \beta}\left[F_{j}\right]_{G}}\right)^{\omega_{j}},
\end{gathered}
$$

whence

$$
\lim _{n \rightarrow \infty} \frac{\beta\left(\frac{1}{p}+\frac{1}{\lambda_{n}} \ln \frac{1}{\left|g_{n}\right|}\right)}{\beta\left(\frac{1}{p}+\frac{1}{\lambda_{n}} \ln \frac{1}{\left|f_{n}\right|}\right)}=\prod_{j=1}^{m}\left(\varrho_{\alpha, \beta}\left[F_{j}\right]_{G}\right)^{\omega_{j}} .
$$

In view of $(9)$

$$
\lambda_{\alpha, \beta}[F]_{G} \geq \varliminf_{n \rightarrow \infty} \frac{\beta\left(\frac{1}{p}+\frac{1}{\lambda_{n}} \ln \frac{1}{\left|g_{n}\right|}\right)}{\beta\left(\frac{1}{p}+\frac{1}{\lambda_{n}} \ln \frac{1}{\left|f_{n}\right|}\right)} .
$$

Therefore, from (10) we obtain

$$
\prod_{j=1}^{m}\left(\varrho_{\alpha, \beta}\left[F_{j}\right]_{G}\right)^{\omega_{j}}=\lambda_{\alpha, \beta}[F]_{G} \leq \varrho_{\alpha, \beta}[F]_{G}=\prod_{j=1}^{m}\left(\varrho_{\alpha, \beta}\left[F_{j}\right]_{G}\right)^{\omega_{j}},
$$

i. e. the function $F$ has generalized regular growth with respect to $G$ and $\varrho_{\alpha, \beta}[F]_{G}=\prod_{j=1}^{m}\left(\varrho_{\alpha, \beta}\left[F_{j}\right]_{G}\right)^{\omega_{j}}$.

\section{REFERENCES}

1. Roy Ch. On the relative order and lower relative order of an entire function// Bull. Cal. Math. Soc. 2010. - V.102, №1. - P. 17-26.

2. Sheremeta M.M. Asymptotic behaviours of entire functions given by power series and Dirichlet series Abstract of thesis of doct. diss. - Kiev, 1987. - 20 p. (in Russian)

3. Kulyavec' L.V., Mulyava O.M. On the growth of a class of entire Dirichlet series// Carpatian Mat. Publ. - 2014. - V.6, №2. - P. 300-309. (in Ukrainian)

Ivan Franko National University of Lviv, Lviv, Ukraine m_m_sheremeta@gmail.com 